\title{
La fabrique de la danse classique : une pratique performative
}

\section{Christine Leroy}

\section{(2) OpenEdition \\ Journals}

Édition électronique

URL : http://journals.openedition.org/danse/1775

DOI : 10.4000/danse. 1775

ISSN : 2275-2293

Éditeur

ACD - Association des Chercheurs en Danse

Référence électronique

Christine Leroy, «La fabrique de la danse classique : une pratique performative », Recherches en danse [En ligne], Focus, mis en ligne le 15 mars 2018, consulté le 19 octobre 2019. URL : http:// journals.openedition.org/danse/1775; DOI : 10.4000/danse.1775

Ce document a été généré automatiquement le 19 octobre 2019.

association des Chercheurs en Danse 


\title{
La fabrique de la danse classique : une pratique performative
}

\author{
Christine Leroy
}

1 L'expression « fabrique de la danse » n'est pas de toute jeunesse : l'idée que la danse se «construit » d'une manière analogue à la pensée et qu'elle ne surgit pas ex nihilo a notamment été affirmée en France par la philosophe Véronique Fabbri ${ }^{1}$. Le terme de «chorégraphie» lui-même indique bien l'idée d'une élaboration réfléchie, par opposition à une "danse» qui serait simple exultation spontanée et immédiate. Le syntagme «fabrique de la danse » est le nom adopté par une start-up parisienne dédiée à la promotion de la jeune création chorégraphique et qui se souhaite «incubateur de talents ${ }^{2}$ »; sans compter bon nombre d'écoles privées, de compagnies ou d'ateliers aux pratiques expérimentales. De façon générale, on admet que «la danse " se fabrique, qu'elle ne résulte pas de la seule inspiration géniale mais qu'elle s'origine dans le matériau-corps.

2 Aussi l'usage du syntagme "fabrique de la danse " renvoie-t-il à une approche progressiste de cet art, par opposition au ballet classique doxiquement considéré comme réactionnaire: tourné vers le passé et des formes narratives et techniques figées, il «ne fabriquerait rien ». La «fabrique de la danse » ainsi entendue comme tournée vers le progrès est certes plurielle, précisément parce qu'il y a autant de chorégraphes " modernes ", " postmodernes ", « contemporains » que de corps; que le hip hop est en évolution permanente, aussi bien que les danses supposément traditionnelles (danses africaines, danses latines) qui suivent autant de procès d'hybridation, que moult styles semblent être nés pour se métamorphoser : breakdance, streetjazz, etc. Les pratiques d'improvisation à finalité performative (Composition en temps réel, Composition instantanée, Tuning Score...), accentuent l'idée d'une «fabrique », puisque les danseurs y sont en même temps les chorégraphes de leur création éphémère. Aussi la chorégraphe allemande Sasha Waltz, qui s'intéresse tout particulièrement aux liens entre espace architectural et corporéité ${ }^{3}$, n'hésite-t-elle pas à affirmer que ses danseurs sont co-chorégraphes de ses pièces ${ }^{4}$. Si la danse s'affirme ainsi comme résultat d'une fabrique, d'une poïsis, c'est par opposition à l'idée d'une 
danse réduite à son spectacle. La danse entendue ainsi n'est pas un spectacle en soi : elle est avant tout un vécu charnel, kinesthésique, psychophysique et centripète. Insister sur la «fabrique » de la danse, c'est promouvoir en même temps son caractère artisanal, sa noblesse en tant que travail sur soi, et c'est précisément faire de ce travail un spectacle en même temps que l'occasion d'une réflexion. La danse n'est, ainsi, pas seulement le résultat d'un processus, mais le processus même d'élaboration chorégraphique. Elle ne se restreint donc pas à la scène, mais se joue, s'auto-génère, se "performe ${ }^{5}$ », en studio, dans l'ipséité $e^{6}$ proprioceptive du corps. C'est la raison pour laquelle beaucoup de chercheurs en danse relèvent le caractère fécond de cette pratique pour la philosophie elle-même ${ }^{7}$ autant que son caractère philosophique à même le corps ${ }^{8}$.

3 La mise en avant d'une telle richesse réflexive du processus créatif en danse permet aux techniques distinctes $\mathrm{du}$ ballet classique, et principalement aux courants dits " contemporains", dans toute leur diversité, de se présenter comme support justifié d'une pensée philosophique du geste dansé, voire du corps en mouvement ${ }^{9}$. La tradition philosophique quant à elle se préoccupe peu de la danse, mais les chercheurs en philosophie, quels que soient leur courant et leur méthodologie (analytique, phénoménologique, esthétique notamment) s'y intéressent de plus en plus ${ }^{10}$. En outre, le champ de la « Recherche en danse » a acquis sa légitimité depuis une bonne décennie en France ${ }^{11}$, conjuguant diverses méthodologies (histoire, sociologie, littérature, sciences cognitives, philosophie, littérature, etc.) pour "penser» la danse. Aucune technique, aucune pratique n'échappe à une telle diversité méthodologique, et les danses tribales autant que le jazz, la «nouvelle danse » d'Isadora Duncan comme les textes de Nijinski sont étudiés au peigne fin par des spécialistes passionnés.

Pourtant, il demeure une dichotomie entre les danses qui « se fabriquent » à partir du corps, qui sont pensables dans le prolongement d'une sorte de " pensée somatique », et les danses qui s'imposent comme techniques aux corps, lesquels doivent s'y plier.

4 La danse classique ou "académique » est de celles-là : malgré la prévalence de l'endehors, ce qui pourrait l'apparenter à des techniques orientales ${ }^{12}$, le ballet classique est considéré, non sans quelque raison, comme technique allant à l'encontre d'un positionnement naturel. De prime abord, sa vocation semble n'être que spectaculaire : la contrainte qu'elle applique aux corps apparaît motivée par l'esthétique seule. Qu'elle le soit, c'est un fait indéniable; mais que la danse classique soit réductible à son esthétique, c'est ce que nous souhaitons récuser.

5 Assurément, et en particulier sous l'impulsion de Pierre Beauchamp puis Jean-Georges Noverre $^{13}$, le ballet classique s'est codifié depuis ses origines et est devenu une technique rigoureuse assortie d'un vocabulaire. Il est particulièrement normé et normatif. Le rôle et sa chorégraphie sont un costume que le danseur endosse et auxquels il doit se conformer. La danse classique ne se fabrique pas, puisque tout est déjà fabriqué ; si l'on observe quelques variations de style suivant les chorégraphies et les chorégraphes, elles sont comparables aux divers accents de ceux qui parlent une même langue, liées à la singularité de l'interprète. Mais ce dernier ne fait que lire et exécuter avec son corps une partition qui lui préexiste et à laquelle il doit se rendre totalement disponible. D'où la réputation, tenace, d'une radicale opposition idéologique entre danses contemporaines et danse classique, laquelle serait d'ailleurs réductible à un seul style; d'où l'idée, surtout, que la danse classique ne pense pas, qu'elle n'est pas réflexive puisqu'elle ne nécessite qu'une exécution. En cela, elle paraît impensable 
philosophiquement. Du moins ne l'a-t-elle que fort rarement été : les "philosophes » qui évoquent la danse classique l'ont systématiquement fait du point de vue de leur regard de spectateur. Bon nombre de danseurs classiques ${ }^{14}$ se sont essayés à l'écriture, mais leur lecture reste restreinte au petit groupe de leurs admirateurs. Certes, la «Recherche en danse " n'évince pas la danse classique : elle l'étudie par le prisme de l'histoire ou le prisme, critique, de la sociologie ${ }^{15}$; celui, bien sûr, de la médecine du sport $^{16}$ et des sciences somatiques ${ }^{17}$. De la sorte, on entérine l'idée que la danse classique a beau s'être transformée en "néoclassique " sous l'impulsion de George Balanchine au $\mathrm{xx}^{\mathrm{e}}$ siècle ${ }^{18}$, elle reste un spectacle avant tout et ne peut pas prétendre à la même légitimité philosophique que les pratiques contemporaines et somatiques. Le formidable travail de Wilfride Piollet ${ }^{19}$, qui a codifié le geste classique d'après les sensations et l'imaginaire du danseur, a néanmoins validé l'idée que la danse classique ne peut se penser qu'à la condition de devenir une danse fondée kinesthésiquement, c'est-à-dire de s'auto-réfuter comme code esthétique ${ }^{20}$. Aussi a-t-on vu disparaître de certains conservatoires les miroirs et les barres, ce qui s'est révélé être une épreuve insurmontable pour certains professeurs ${ }^{21}$. Or, on ne peut pas non plus nier l'origine et l'intention spectaculaire de la danse classique: elle semble bien tendue entre spectacularité esthétique et vécu kinesthésique. C'est pourquoi il nous semble nécessaire de penser cette tension; et nous proposons de le faire dans les termes d'une performativitée ${ }^{22}$, c'est-à-dire d'une production coïncidant avec le processus même de production; d'une « fabrique ».

6 Nous postulerons ici la singularité en même temps que la nécessité d'une pensée philosophique de la danse classique: quoiqu'il ne semble pas exister à proprement parler de "philosophie de la danse classique", hormis dans les termes d'un essentialisme fondé sur le seul spectacle de la danse - autrement dit, loin du danser -, la danse (néo)classique ${ }^{23}$ nous semble résulter d'un processus de création tout aussi complexe que la danse contemporaine, et cependant très singulier. Si la technique classique est une contrainte du naturel au même titre que toute technique, c'est aussi d'elle que le corps se joue lorsqu'il danse; nous soutenons ainsi que la technique classique n'a pas de visée seulement spectaculaire. Autrement dit, en danse classique, la fabrique se joue dans ce qui ne se voit pas, dans ce qui se vit, de sorte qu'un geste n'est pas l'endossement d'une figure, mais au contraire le résultat de toutes celles qui ont été éliminées. Le danseur cherche non à effectuer des figures, mais à dépasser le caractère contraignant des normes académiques; dans une certaine mesure, il cherche à se délester du caractère d'effort que constitue l'effectuation du mouvement classique. Autrement dit, la danse (néo)classique se joue non dans les positions classiques, mais entre les positions, dans le mouvement qui lie l'une à l'autre et dont elles sont des étapes ou passages obligés. Si la danse est bien, de façon générale, une dynamique et un flux kinésique, la danse (néo)classique n'échappe pas à ce grand principe. Or, tout mouvement peut être dit " performatif ", au sens où il est dans son écriture, et n'est pas une résultante réifiée à l'instar d'un produit. Le terme de "chorégraphie", qui s'applique indistinctement à tout type de danse, souligne bien le caractère scriptural graphique - du geste dansé, mais ce geste s'évanouit dès qu'il n'est plus effectué. On peut bien filmer la danse, elle n'est plus sous sa forme physiquement incarnée. Nous affirmons que la danse classique n'échappe pas, loin s'en faut, au caractère performatif de la danse. Elle peut bien faire l'objet d'une identification et d'une formalisation verbale (" pas de bourrée, saut de chat, glissade, grand jeté !»), mais la danse excède ce par quoi l'on nomme chaque type de mouvement ou de figure - elle rejoint d'ailleurs en 
cela les autres pratiques chorégraphiques. Le danseur classique n'est pas un technicien qui agit une partition, il n'" incarne " pas un mouvement qui existerait absolument, hors du corps. Le mouvement classique est performatif : il se crée dans le corps du danseur. Nous récusons ce faisant tout réalisme des formes en danse classique : le pas, la figure, le mouvement n'existent pas en dehors de leur effectuation. S'il y existe certes un vocabulaire et des concepts, de type "temps levé », "pirouette " et autres "grands battements", il ne nous semble pas légitime ni opérant de réifier de tels concepts. En tant que concepts, ils relèvent d'une projection de la part d'un maître de ballet, d'un chorégraphe ou d'un professeur de danse, sur le corps de l'interprète qu'il s'agit de plier à une forme, et éventuellement aussi d'une opération d'abstraction de la part de l'apprenti danseur; mais le concept n'est pas la danse, qui se joue à même le corps de l'interprète, à l'occasion d'un mouvement marqué spatio-temporellement. C'est aussi pourquoi la connaissance des concepts ou la lecture d'un traité de danse classique ne saurait faire connaître le danser. C'est enfin ce qui justifie notre assertion : la danse classique ne préexiste pas à sa fabrique vécue et performative.

7 Aussi avancerons-nous ici l'idée que, contrairement à une opinion trop répandue y compris dans le milieu de la recherche en danse, il existe une «fabrique performative de la danse (néo)classique » bien spécifique, ce qui légitime hautement d'initier une phénoménologie du ballet à partir des corps dansants. Nous souhaitons ici poser les linéaments d'une réflexion à ce sujet, avec tout ce que peut présenter de balbutiant un début de questionnement.

\section{Des idées reçues et solidement ancrées dans les esprits. Mais pas dans les corps}

8 Lorsque l'on assiste à un ballet classique à l'Opéra de Paris, lorsque l'on observe Sylvie Guillem dans le ballet Paquita ${ }^{24}$, on constate l'effectuation parfaitement maîtrisée, par le danseur ou la danseuse, d'un geste relevant du vocabulaire classique. Aucune hésitation dans le pas: il y a virtuosité technique. La "pirouette» par laquelle la danseuse effectue un tour sur pointes, telle une toupie, est géométriquement conçue : qu'elle fasse " un tour ", "deux tours", "trois tours ", elle n'en fera pas " un trente-deux dixièmes ». Le grand écart est à cent quatre-vingts degrés. Tout semble, et tout est, sous contrôle. On en viendrait ainsi à penser que ce pas est acquis, une bonne fois pour toutes. De même qu'un mot écrit sur du papier y demeure une fois l'écriture achevée, de même le pas effectué en danse classique peut passer pour «acquis » par le corps, compris définitivement.

En réalité, il n'en est rien. Qu'il suffise pour ce faire de quitter des yeux la scène, et d'explorer la réalité du studio. Ici, on ne fait pas que transpirer : on rate. On échoue. On retravaille le geste. La perfection technique qui se donne à voir en spectacle n'est que pour le regard : en réalité, elle est toujours à l'horizon du corps du danseur ou de la danseuse, sans qu'il ou elle puisse assurer absolument de "l'avoir dans les jambes ». Il faut toujours recommencer et un peu de fatigue au réveil, quelques semaines sans pratique auront raison d'un corps qui jamais n'acquiert la danse classique. Ce qu'il y a lieu de comprendre, c'est véritablement que la danse classique se joue à même le corps, dans un vivre kinesthésique et proprioceptif réels et perpétuellement remaniés. Si les pas ont été codifiés sous la forme d'un vocabulaire, il ne faut pas céder à la tentation essentialiste et penser qu'ils existent indépendamment des corps. Assurément il existe 
en danse classique tout particulièrement une «forme » ou « idée ", un mouvement dit « idéal » ou " parfait »; mais il s'agit en cela d'un concept au sens platonicien d'eidos, c'est-à-dire une représentation mentale abstraite et par nature désincarnée. Il ne saurait s'agir alors d'un danser, c'est-à-dire d'un vécu immanent. « Rater » sa pirouette, ce n'est pas échouer dans l'adéquation d'un mouvement effectué à un idéal esthétique et spectaculaire à atteindre, qui préexisterait au mouvement, mais plutôt ne pas parvenir à créer, à faire émerger et somme toute à fabriquer une pirouette : ce qui se joue dans l'effectuation du mouvement, c'est une sensation de légèreté, de virevolte qu'aucun dessin extérieur pas plus que le moindre concept ne sauraient exprimer adéquatement. La danse classique se joue dans l'immanence du mouvement, à l'intérieur des corps. Si le spectateur croit voir la danse classique sur le corps de l'interprète, il ignore toute une réalité kinesthésique et psychomotrice fondamentale. C'est dans cette réalité kinesthésique que se fabrique la danse classique en tant que performance.

Ce qui résulte de cette affirmation, et qui s'oppose à un second a priori, c'est que contrairement à un fantasme romantique tenace, la danse classique ancre celui ou celle qui la pratique dans la réalité des corps et de la matière. La sylphide éthérée des ballets romantiques, la danseuse ailée, sont données comme telles pour le spectateur, dans le contexte d'une narration fantasmagorique. Dans son journal intime ${ }^{25}$, Anna Pavlova explique qu'il lui faut être la plus légère possible, et l'on attend de la danseuse classique autant que du danseur de manifester légèreté, grâce, subtilité et spiritualité par la médiation du corps. Mais on se cantonne alors à la seule narration. Il ne s'agit pas de danse classique, mais d'imaginaire narratif et spectaculaire. Sur scène, la danseuse semble aérienne; dans son corps, elle s'amarre au socle terrestre. Il ne s'agit pas de récuser l'idée que la danse classique est, aussi, spectaculaire ; mais la façon dont elle se vit et s'éprouve, la performativité dont elle relève est sans commune mesure avec le spectacle qu'elle offre.

11 Ainsi, pour Anna Pavlova elle-même, le corps s'impose en permanence, dans le vécu de la pesanteur. Pour le danseur classique, le saut se prépare dans le sol et son amplitude n'est garantie que par l'ancrage dans ce socle terrestre. À l'instar de tout athlète de haut niveau, les danseurs classiques sont particulièrement à l'écoute de leur corps, duquel ils bravent souvent les signaux de douleur, mais qui reste la matière première de leur pratique et le medium de leur art - au sens premier d'artisanat, d'un faire gestuel autographique ${ }^{26}$. La danse classique comme pratique et comme fabrique n'est donc en rien une réprobation de la réalité des corps et de la pesanteur matérielle : elle y renvoie en permanence, comme condition contraignante mais nécessaire. Le pied, charnière entre le sol et le corps, y fait l'objet d'un soin particulier. Il semble y avoir une incommensurabilité entre ce qui se voit de la danse classique, en particulier la grâce qui se dégage de son spectacle et la façon dont les gestes aériens y subliment la pesanteur charnelle, et le vécu réel du danseur : le corps est là, charnel, pesant, jubilant et parfois souffrant, toujours ressentant.

Il nous faut dès lors conclure cette remise en question de deux a priori majeurs du spectateur de danse classique. Nous pourrons ainsi, à partir de ce renversement, proposer un nouveau « regard » sur la danse classique, à partir de sa fabrique et non à partir de son spectacle. Fabrique et spectacle ne sauraient être antinomiques, mais il nous semble que la première n'a pas été suffisamment considérée - le ballet classique ayant été le plus souvent réduit à sa dimension spectaculaire et à ses œuvres, quand nous souhaitons nous intéresser à l'œuvrer lui-même. 
Loin de n'être que le résultat spectaculaire de l'effectuation d'un pas pré-donné, la danse classique est un travail de la matière-corps, dans le milieu terrestre et dans un système régi par la pesanteur. Il y a lieu de considérer ce travail indépendamment de tout processus narratif ou fictionnel. «Faire de la danse classique », ce n'est pas créer un produit narratif allographique, c'est-à-dire distinct de l'interprète : c'est, toujours, interpréter, c'est-à-dire performer un geste qui ne survit pas à son vécu kinesthésique. Autrement dit, une approche phénoménologique ${ }^{27}$ et kinesthésique, au sens husserlien ${ }^{28}$, s'impose dès lors que l'on assume le versant kinesthésique performatif du geste classique : il s'agit de questionner le «comment » du geste, le vécu ${ }^{29} \mathrm{du}$ danseur ou de la danseuse, et de le dissocier de son «donné " phénoménal à autrui. C'est bien dans ce "comment" que se joue, à nos yeux, le caractère performatif de la danse classique. C'est aussi là que se joue son potentiel créatif et le surgissement d'un geste « néo » classique que nous évoquerons plus bas dans sa spécificité.

Une prise de recul face au résultat de notre réflexion nous conduit à la question suivante : si la danse classique est certes tout à la fois performance kinesthésique et spectacle, présentant en cela une ambiguïté que trop de discours sur la danse classique semblent taire, ne va-t-on pas trop loin dans la mise entre parenthèses de la spectacularité de la danse classique, en nous focalisant sur la subjectivité du danseur/ de la danseuse?

En effet, on ne peut pas nier le projet esthétique de la chorégraphie classique : il faut donner à voir quelque chose. Les miroirs dans les studios de danse se substituent ainsi aux regards des spectateurs, tout en rappelant l'objectif d'une telle pratique: le spectacle. En cela, la danse classique se distingue du champ très vaste de la danse contemporaine : si la majorité des danseurs et danseuses classiques amateurs n'a pas pour projet d'intégrer une compagnie et de se donner en spectacle, la pratique de la danse classique elle-même conserve un caractère narcissique indubitable: elle s'effectue traditionnellement face à un miroir, auquel les pratiquants s'amarrent autant, si ce n'est davantage, qu'à la barre. Lorsque le danseur n'est pas regardé, jugé par autrui, il lui faut pouvoir s'évaluer, poser sur lui-même un regard critique de spectateur. À l'opposé, bien des pratiques en danse contemporaine ne sont pas subordonnées au regard du spectateur, et le plus souvent les studios où l'on pratique les danses contemporaines se dispensent très bien de miroir. L'on s'y préoccupe en premier lieu de la proprioception et de l'écoute à soi, ce qui constitue un point de départ du mouvement. Autrement dit, la cause du mouvement contemporain semble être une cause motrice et non une cause finale ${ }^{30}$ comme en danse classique.

Il ne s'agit pas pour nous de récuser le caractère spectaculaire de la pratique de la danse classique. La danse classique est bel et bien codée esthétiquement, et liée à un idéal gestuel désigné par les termes de son vocabulaire et les normes de son esthétique. Ce qu'il s'agit pour nous d'affirmer, c'est qu'à cette esthétique identifiable par un vocabulaire se joint un vécu kinesthésique et charnel en lequel se joue « la fabrique de la danse classique ». Nous employons le terme de "jeu " au sens où Donald Woods Winnicott l'emploie dans son ouvrage Jeu et Réalitée ${ }^{31}$ pour désigner l'espace de mouvement possible au sein d'un mécanisme. On parle ainsi du «jeu» d'une poignée sur une porte, du « jeu » d'une articulation, ou l'on dira d'une vis trop serrée sur son écrou qu'elle «interdit tout jeu ». La Recherche en danse, du moins pour partie, s'intéresse véritablement à la kinesthésie du geste contemporain : il est admis que la danse contemporaine est la manifestation esthétisée d'un pré-mouvement ${ }^{32}$ du corps. 
Concernant la danse classique, il serait faux d'affirmer que l'en-dehors ou le "fouetté » "partent du corps » et ne sont que l'expression d'un mouvement spontané : bien sûr, l'effectuation de ces mouvements requiert une grande technicité, structurée sous la forme d'un vocabulaire. Mais c'est justement ce qui permet à la danse classique de pouvoir être considérée comme performative : elle se joue non dans ses mots - les pas, les positions - et ses phrases - les combinaisons de pas -, mais entre les mots, derrière eux, dans le jeu entre la subjectivité kinesthésique psychophysique du danseur, le vécu et le projet du chorégraphe et/ou du maître de ballet, et ce code préexistant qu'est la technique. C'est pourquoi nous soutenons que, loin de se réduire à sa spectacularité, la danse classique résulte d'un jeu autour du code, articulé entre danseur et réflecteur - le spectateur--, des usages faits du corps pour se jouer de ce code esthétique et de ce regard réfléchissant, et qu'elle se performe ainsi dans ce cheminement entre les pas et positions, entre les normes académiques, entre celui qui reçoit et celui qui se joue. Ce postulat nous permet, du reste, de rassembler ce qui pourrait paraître antinomique : le spectateur "joue " un rôle dans ce jeu performatif, puisqu'il y exerce la fonction de regard réfléchissant au sujet dansant son propre vécu kinesthésique, mieux qu'un miroir. Si la danse classique est bien spectaculaire, c'est parce que sa spécularité y officie à titre de réflecteur kinesthésique. Nous voulons dire par là que l'apparence de la danse classique n'est que l'expression, forcément superficielle, donc partielle, d'un vécu kinesthésique. De là, il n'est pas impossible de penser la fondation de l'empathie kinesthésique, éprouvée par le spectateur de danse classique, dans le vécu kinesthésique même du danseur. Cette hypothèse s'inscrit dans la continuité des travaux de Hubert Godard et de la phénoménologie merleau-pontienne de la motricité 33 .

Nous affirmons ainsi la réalité kinesthésique d'une "fabrique de la danse classique ", dans le sens d'un tel «jeu» entre code et corps, vécus phénoménologique et kinesthésique à la fois du danseur et du spectateur. Elle nous semble tout particulièrement à l'œuvre aujourd'hui dans le courant dit « néoclassique ».

\section{Le travail de la danse classique : le studio}

«Sans cesse sur le métier remettre son ouvrage... ».

L'image d'Épinal nous présente un danseur ou une danseuse qui "répète quotidiennement ses exercices à la barre ». Il ne s'agit pas d'une image erronée. Ce qui l'est en revanche, c'est la présentation d'une telle démarche comme une "répétition " relativement ennuyeuse, fastidieuse et rébarbative. À ce sujet, il y a erreur: si les exercices à la barre étaient une répétition monotone, il y a longtemps qu'on leur aurait substitué d'autres moyens de s'échauffer. L'erreur consiste à croire que la barre est un échauffement, à l'instar des exercices abdominaux et des pompes destinés à gainer le corps du sprinter. Les exercices abdominaux ne sont pas la course à pied; mais les exercices à la barre sont déjà de la danse classique. L'enchaînement est proposé par l'enseignant et il s'agit bel et bien de suivre une "routine", dont chaque instant est codifié, dont chaque pas est une étape obligée à respecter. On ne passe pas en "grand plié » sans être passé par l'étape du "demi-plié », on ne s'élève pas sur pointes en sautant car l'étape de la demi-pointe en est un intermédiaire nécessaire et convenu. En quoi ce constat corrobore-t-il notre affirmation du caractère "créatif» et " performatif » de la danse classique? Ne sommes-nous pas ici en train de récuser ce que nous avons tâché de mettre en évidence jusqu'à présent? 
17 Le rôle des exercices à la barre est d'améliorer et de consolider «le placement »: autrement dit, l'en-dehors de tout mouvement du danseur. Il s'agit bel et bien d'exercice, sorte de gymnastique du positionnement adéquat. Mais ce faisant, le danseur et la danseuse expérimentent des sensations : celles du «bon placement ». Ce sont ces sensations kinesthésiques liées à l'en-dehors qu'il s'agira de réitérer lorsqu'il ou elle se retrouvera " au milieu ", sans l'appui de la barre. Comme dans toute pratique sportive, le temps préparatoire de « la barre » est aussi celui d'une prise de conscience de la physicalité ; mais la spécificité de « la barre » consiste dans l'expérimentation du "placement ", c'est-à-dire le juste positionnement dans l'espace pour relever le défi de l'en-dehors. Ce n'est pas simple : il faut trouver dans sa propre expérience kinesthésique celle de l'en-dehors au niveau du bassin, et tâcher de la prolonger jusque dans le pied pour la réitérer à chaque instant.

excice initie ce qui sera le fil conducteur de toute la pratique de la danse classique. Il s'agira en permanence de défier la pesanteur, de déjouer les obstacles techniques, non pas en les fuyant, mais en les surmontant. De fait, les contraintes imposées par l'esthétique académique du ballet sont autant de points de repère ; elles sont riches de jeu, donc de danse. Recherche créative d'échappatoires dansantes, le ballet prend un sens kinesthésique nouveau qui échappe à son spectateur. Ce sens kinesthésique est celui d'une performance au sens de John Austin $^{34}$ : à mesure que le danseur recherche le libre «jeu » malgré la contrainte de l'en-dehors, il performe une danse classique. Cette "fabrique» au présent n'est donc pas seulement un produit esthétique visible, mais aussi dans le même temps et peut-être avant tout un vécu kinesthésique singulier. C'est pourquoi la contrainte de l'en-dehors est essentielle, quitte à être subvertie : elle génère un certain type de gestuelle, donc une esthétique en même temps qu'un vécu kinesthésique singulier. La mobilisation d'une contrainte " de jeu " ne distingue pas la danse classique des autres formes de danse, qui recourent souvent à ce type de procédé pour créer du jeu et de la danse. Quand le danseur contemporain ou le performer doit, par exemple, ne mouvoir que son poignet et générer la danse à partir de celui-ci, le danseur classique doit répondre aux exigences de l'endehors et de l'extension de la pointe des pieds. Loin s'en faut, dès lors, que la danse classique s'impose aux corps par opposition aux pratiques de danse contemporaine «à partir» des corps: ce qui s'impose au danseur classique, comme au danseur contemporain, ce sont certaines contraintes qui initient la créativité par la manière dont on s'en joue et dont on les contourne. Aussi est-il possible d'affirmer que la fabrique de la danse classique part du corps, qu'elle en émerge comme un excès. La pièce Amelia $^{35}$, de la compagnie La La La Human Steps, nous semble en fournir une illustration : dans une salle carrée manifestement sans issue, un homme fait tourner entre ses mains une danseuse sur pointes. La virtuosité technique est de mise, mais la chorégraphie d'Édouard Lock n'est que succession répétitive de figures imposées dérivées de la " pirouette », de l'« atttitude », de l'« arabesque » et de la « fente ». La danseuse semble être contrainte, d'une part par son partenaire qui lui impose un mouvement giratoire, d'autre part par les pointes qui restreignent la surface au sol de ses pieds et la condamnent au mouvement d'une toupie. Lorsqu'elle descend des pointes et s'éloigne du danseur en marche naturellement, elle reste comme attirée inexorablement par son partenaire sur lequel elle se projette à nouveau et entre les mains duquel elle renouvelle les figures imposées. La contrainte d'une faible diversité de mouvements aliène la danseuse, qui faute de pouvoir s'en libérer tente de les excéder : comme c'est souvent le cas en danse néoclassique, le cou de pied est poussé à l'extrême dans 
l'inclinaison du corps, les pirouettes sont enchaînées à l'infini et le plus rapidement possible. Il nous semble bien que la performance se joue précisément dans la marge que crée la danseuse à partir des figures imposées, et le désir de liberté dont elle témoigne. Aussi nous apparaît-il désormais nécessaire d'explorer l'hypothèse d'une fabrique de la danse néoclassique, non sur ou avec des corps, mais à partir du désir même des corps.

\section{La création en danse classique : le « néoclassique »}

Il serait inutile de retracer ici l'histoire du style néoclassique, non qu'elle soit universellement connue, mais parce qu'elle serait trop longue à relater. Nous renvoyons à ce sujet à l'excellent article, appuyé d'extraits vidéos, de Laëtitia Basselier au sein de la revue numérique Danse avec la plume ${ }^{36}$. Ce qu'il faut surtout avoir présent à l'esprit, c'est que la danse «néoclassique » a fait le pari de la dissidence à l'égard du code académique, sans le réfuter comme l'ont fait les danseuses dites "modernes » (Isadora Duncan ou Martha Graham). C'eût pu n'être qu'un vœu pieux, comme le sont bon nombre d'ambitions de rénovation et de renouvellement stylistiques. Mais il y a bel et bien eu rupture avec le style académique systématisé par Beauchamp et Noverre; une rupture paradoxalement «en continuité ». Nous employons à dessein le terme de "rupture», car il désigne une réalité gestuelle. Le style néoclassique a ceci de spécifique qu'il alterne l'en-dehors et l'en-dedans. Il admet l'excès du mouvement jusqu'au déséquilibre, c'est-à-dire jusqu'à la chute. En d'autres termes, il excède la ligne et la sphère parfaites, et introduit une véritable rupture géométrique à travers les angles et l'imperfection - la chute.

Il résulte un certain «style " de cette volonté de " rupture en continuité ", propre aux chorégraphes néoclassiques, dont les États-Unis ont été une terre très féconde mais pas exclusive $^{37}$. Or, il semble bien que ce style souligne ce que nous avons avancé jusqu'ici : le jeu avec le code, au sein même du cadre constitué par la danse "classique "; mais un jeu qui fait lui-même spectacle. L'art du style néoclassique consiste ainsi à mettre en avant et à donner à voir toutes les manières dont on peut subvertir le requisit de l'endehors et de l'extension du pied en pointe. La chorégraphie y pousse la technique audelà de ce qu'elle exige ; le corps virtuose y excède, au nom de la technique, les limites qu'elle détermine. Aussi la chorégraphie néoclassique donne-t-elle en spectacle cet excès de la technicité sur la technique elle-même.

Ainsi nous semble-t-il que la danse néoclassique en particulier exploite le potentiel performatif de la danse classique. On crée de la danse néoclassique comme on écrit un roman: il faut maitriser le vocabulaire, il faut disposer des outils linguistiques nécessaires pour donner sens à l'enchaînement des mots; il faut posséder l'art de faire passer le fond à travers la forme, l'art de faire surgir le fond par la forme elle-même aussi. Mais il faut avant tout savoir créer, c'est-à-dire savoir trouver du jeu entre les mots, entre les lignes, derrière les phrases elles-mêmes, et initier chez le lecteur l'élaboration d'un champ mental et imaginaire plus vaste encore.

En outre, la danse néoclassique s'écrit à même le corps, à partir de lui plutôt que sur lui, à partir de l'articulation entre kinesthésie et système normé; elle en est le jeu spectaculaire. La danse néoclassique se fabrique comme pratique performative : l'épochè de sa dimension spectaculaire permet d'en révéler la dimension kinesthésique première, génératrice d'une gestuelle et d'une esthétique, et singularisée dans les corps de l'interprète et du chorégraphe. 
Lorsque Husserl en effet inaugure une telle démarche épochale ${ }^{38}$, ce n'est pas pour réfuter l'existence du monde mais pour mieux comprendre comment le sujet constitue ce monde phénoménal en même temps qu'il se constitue lui-même comme existant. De manière analogue, l'épochè de la dimension spectaculaire de la danse néoclassique peut permettre d'étudier la manière dont elle se performe, dans l'entrelacs entre contrainte posturale de l'en-dehors et liberté motrice. Si la Recherche en danse contemporaine a trouvé des échos certains dans une telle démarche phénoménologique ${ }^{39}$, il y a lieu de tester la fécondité de la phénoménologie à propos de la danse néoclassique. Il n'est pas à exclure que par un effet retour, les spécificités performatives de la danse néoclassique apportent un éclairage nouveau au concept de « kinesthèse " chez Husserl, à celui de « chair » chez Merleau-Ponty ou plus largement à la phénoménologie esthétique.

\section{BIBLIOGRAPHIE}

AUSTIN John Langshaw, Quand dire c'est faire [1962], trad. Gilles Lane, Paris, Éditions du Seuil, 1970 BALLANFAT Elsa, La Traversée du corps. Regard philosophique sur la danse, Paris, Hermann, 2015.

BASSELIER Laëtitia, « Une histoire du (néo)classique en dix ballets », revue électronique Danses avec la plume, 12 février 2015, http://www.dansesaveclaplume.com/en-coulisse/une-histoire-duneoclassique-en-10-ballets/. Consulté le 31 octobre 2017.

BASSELIER Laëtitia, « Comment construire une (des) philosophie(s) de la danse classique ? ", communication à la journée « Pratiques de thèse en danse » de l'atelier des doctorant.e.s du CND, http://isis.cnd.fr/IMG/pdf/atelier_2017_fevrier.pdf. Consulté le 12 février 2018.

BASSELIER Laëtitia, «Étienne Souriau et la danse, rencontres (manquées ?) », in Nouvelle Revue d'Esthétique, 2017/1 ( ${ }^{\circ}$ 19), https://www.cairn.info/revue-nouvelle-revue-d-esthetique-2017-1p-85.htm. Consulté le 12 février 2018.

BASSELIER Laëtitia, « Penser le classique », in Marius Petipa. Étoilement d'une cuvre, exposition au CND (livret d'exposition), Pantin, CND, 2018.

BIGÉ Romain, « Ce que la phénoménologie peut apprendre de la danse. Straus, Merleau-Ponty, Patočka », Recherches en danse [En ligne], 5 | 2016, mis en ligne le 15 décembre 2016. Consulté le 31 octobre 2017. URL : http://danse.revues.org/1394.

FABBRI Véronique Fabbri, Danse et Philosophie. Une Pensée en construction, Paris, L'Harmattan, «Esthétiques », 2007.

GIOFFREDI Paule, «Phénoménologie de la danse contemporaine », Recherches en danse [En ligne], 1 | 2014, mis en ligne le 01 mars 2013. Consulté le 31 octobre 2017. URL : http://danse.revues.org/ 596.

GODARD Hubert, « Le geste et sa perception », in MICHEL Marcelle, GINOT Isabelle, La Danse au XXe siècle, Paris, Éditions Bordas, 1995, pp. 224-229. 
GRECIET Cheryl, Évolutions et nouveaux enjeux de la théâtralité en danse contemporaine : l'exemple de François Verret et de Sasha Waltz, Thèse de Doctorat, sous la direction de Didier Plassard, Université de Rennes, 2005.

HARDY Jean-Sébastien, Phénoménologie des kinesthèses et Ontologie du geste : Constitutions originaires du monde et de la chair chez Husserl, thèse de doctorat en Philosophie, sous la direction de Jean-Luc Marion, Paris-Sorbonne/Paris IV et Université de Laval, 2014. www.theses.ulaval.ca/ 2014/30866/30866.pdf.

HUSSERL Edmund, La Crise des sciences européennes et la Phénoménologie transcendantale (1936), trad. Gérard Granel, Paris, Gallimard, 2004.

LALLIER Joël, Entrer dans la danse, Paris, CNRS Éditions, 2017.

MERLEAU-PONTY Maurice, Phénoménologie de la perception (1945), Paris, Éditions Gallimard, 1976.

NICOT Isabelle, Tirer son épingle du jeu : danse classique et construction sociale des figures de la féminité. Études sur le genre, Thèse de doctorat, sous la direction de Marina Honta, Université de Bordeaux, 2016. https://tel.archives-ouvertes.fr/tel-01652132v2/document.

NOVERRE Jean-Georges, Lettres sur la danse [1760], Paris, Hachette Livre BNF, 2012.

Revue Médecine des arts, $\mathrm{n}^{\circ}$ 83, Spécial danse « Santé des artistes, santé du danseur », 2017.

VAN DYK Katharina, «Sentir, s'extasier, danser », Implications philosophiques [En ligne], première partie mise en ligne le 19 juin 2010 et deuxième partie mise en ligne le 21 juin 2010. Consulté le 31 octobre 2017. URL : http://www.implications-philosophiques.org/langage-et-esthetique/ implications-de-la-perception/sentir-s\%E2\%80\%99extasier-danser-1/ et http:// www.implications-philosophiques.org/langage-et-esthetique/implications-de-la-perception/ sentir-s\%E2\%80\%99extasier-danser-2/.

WINNICOTT Donald Woods, Jeu et Réalité (1971), trad. Claude Monod, Jean-Baptiste Pontalis, Paris, Folio Essais, 2015.

\section{NOTES}

1. FABBRI Véronique, Danse et Philosophie. Une Pensée en construction, Paris, L'Harmattan, «Esthétiques », 2007.

2. Le "Centquatre-Paris » et le Carreau du Temple hébergent cette structure d'aide à la jeune création chorégraphique. Voir http://www.lafabriquedeladanse.fr/, page consultée le 31 octobre 2017.

3. La chorégraphe allemande utilise fréquemment l'espace comme contrainte motrice et gestuelle. Fille d'architectes, son travail a toujours accordé une place majeure à l'espace scénique comme outil chorégraphique. Voir le site de la compagnie : https://www.sashawaltz.de/uebersasha-waltz-guests/, page consultée le 31 octobre 2017.

4. Affirmation extraite d'un des entretiens réalisés par GRECIET Chéryl au sein de sa thèse de doctorat en Art Évolutions et nouveaux enjeux de la théâtralité en danse contemporaine : l'exemple de François Verret et de Sasha Waltz, sous la direction de Didier Plassard, Université de Rennes, 2005.

5. Le verbe "performer" est un emprunt à l'anglais to perform. Ce qui ne saurait manquer de susciter l'étonnement, c'est qu'il désigne initialement et avant tout le fait d'agir. Celui qui "performe ", c'est celui qui agit: l'acteur. On retrouve en anglais la polysémie française du mot " acteur ", de manière différente : puisque les acteurs, cette fois au sens de "comédiens ", sont sur scène, ce que nous désignons en français par « Arts de la scène » prend en anglais le nom de Performing Arts. Or, en Philosophie, le verbe to perform renvoie également à la philosophie du 
langage : dans Quand dire, c'est faire (1962, trad. Gilles Lane, Paris, Éditions du Seuil, 1970), Austin

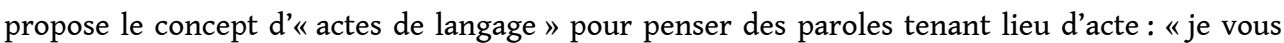
condamne à une peine de...", «je vous déclare mari et femme», etc. Enfin, le terme de "performance » a été utilisé dans le champ artistique durant la première moitié du $\mathrm{xx}^{\mathrm{e}}$ siècle et jusqu'à nos jours, pour désigner des « actions » faites par des artistes hors espace scénique (rue, commerce, musée...), uniques, éphémères. Le terme de « performance » se révélait plus approprié que celui de "show ", puisqu'il renvoyait d'une part à l'idée d'action, d'autre part à l'idée qu'une telle action se faisait en même temps spectacle (performing art) au fil de son effectuation imprévisible, et en même temps à ce « dire » qui est un « faire » artistique. Aujourd'hui, le verbe "performer» semble être passé dans le langage artistique car il est moins ambigu que le verbe «acter »; mais il n'est pas d'emploi courant dans le langage ordinaire, d'où ce rappel historique sur un terme qui n'a rien de commun avec le fait de «faire une performance » sportive par exemple.

6. Le mot « ipséité » relève du vocable phénoménologique husserlien. En latin, ipse signifie « soimême »: il a valeur de pronom réfléchi. Le mot «ipséité » présente l'avantage de désigner la réflexivité au sens propre, sans présenter l'ambiguïté du mot "réflexivité » associée par la tradition philosophique à l'esprit et non au corps.

7. Voir notamment BALLANFAT Elsa, La Traversée du corps. Regard philosophique sur la danse, Paris, Hermann, 2015.

8. La section «danse » de l'Université Paris VIII, fondée par Michel Bernard et Hubert Godard, promeut l'idée d'une pensée critique fondée dans la pratique de la danse, par opposition à une approche philosophique traditionnelle qui ne ferait de «la danse » qu'un objet esthétique comme un autre.

9. Certaines pratiques de la danse contemporaine se voient qualifiées de "non danse », sans que l'on sache bien définir vraiment la limite entre « danse » et « non danse ».

10. Pensons notamment à Maxine Sheets-Johnstone, Michel Bernard, Laurence Louppe et Renaud Barbaras.

11. L'Association des Chercheurs en danse a été créée en 2007, même si la « Recherche en danse » lui a évidemment préexisté.

12. Nous pensons notamment à la figure du lotus dans le yoga, à l'analogie possible entre posture de l'arbre et retiré en danse classique, ainsi qu'aux paumes de main jointes sur la tête dans les bras en couronne; nous pensons également aux danses traditionnelles indiennes, où l'on retrouve l'en-dehors caractéristique de la danse classique. Toutefois, nos recherches sur une éventuelle inspiration orientale à l'origine de la danse classique ne permettent pas pour le moment d'établir de lien entre ces deux pratiques culturellement distinctes.

13. Voir NOVERRE Jean-Georges, Lettres sur la danse [1760], Paris, Hachette Livre BNF, 2012.

14. Nous pensons notamment, pour les plus connus d'entre eux, à Claude Bessy, Maurice Béjart, Roland Petit, Nicolas Leriche... entre autres.

15. Voir notamment LALLIER Joël, Entrer dans la danse, Paris, CNRS Éditions, 2017. Voir également NICOT Isabelle, Tirer son épingle du jeu : danse classique et construction sociale des figures de la féminité. Études sur le genre, Thèse de doctorat, sous la direction de Marina Honta, Université de Bordeaux, 2016.

16. Voir Revue Médecine des arts, $n^{\circ} 83$, Spécial danse « Santé des artistes, santé du danseur », 2017. 17. Il nous apparaît également indispensable de mentionner le travail en cours de Laëtitia Basselier, actuellement en doctorat de Philosophie de la danse à l'Université de Lille III sur « la catégorie de danse classique et la création chorégraphique (néo)classique en France de 1945 à la fin des années $1960 »$. Voir BASSELIER Laëtitia, « Comment construire une (des) philosophie(s) de la danse classique ? ", communication à la journée « Pratiques de thèse en danse » de l'atelier des doctorant.e.s du CND, http://isis.cnd.fr/IMG/pdf/atelier_2017_fevrier.pdf, page consultée le 12 février 2018. Voir également «Étienne Souriau et la danse, rencontres (manquées ?) », in Nouvelle 
Revue d'Esthétique, 2017/1 ( $n^{\circ} 19$ ), <en ligne>, https://www.cairn.info/revue-nouvelle-revue-desthetique-2017-1-p-85.htm, page consultée le 12 février 2018, et « Penser le classique », in Marius Petipa. Etoilement d'une œuvre, exposition au CND (livret d'exposition), Pantin, CND, 2018.

18. Né en Géorgie en 1904, le danseur a intégré les Ballets Russes de Diaghilev avant d'émigrer aux États-Unis pour y fonder le New York City Ballet. Là, il a procédé à une refonte de la technique classique, lui insufflant une licence dont elle était dépourvue et introduisant, entre autres, l'en-dedans comme posture légitime et le fléchissement du pied au niveau de la cheville comme alternative possible à l'extension sur pointes.

19. L'ancienne danseuse étoile, décédée en 2015 , s'est investie dans la recherche pédagogique une fois sa carrière de danseuse finie. Elle a élaboré un système proprioceptif profitable aux danseurs classiques, basé sur un imaginaire anatomique intitulé « les barres flexibles ».

Pour plus d'information, voir le site http://www.clefdesole.fr/accueil_fr.html, page consultée le 31 octobre 2017.

20. En particulier, les cours de barre flexible refusent le miroir comme repère visuel autocorrectif pour le danseur, le studio de danse est dépourvu de barres tangibles. L'enseignement est fondé sur la découverte de repères internes, proprioceptifs et anatomiques, par le danseur. Ce qui importe n'est pas le résultat esthétique (« est-ce que je fais bien la figure que je souhaite effectuer?») mais le ressenti intime (« est-ce que je ressens adéquatement ce que je souhaite ressentir?»).

21. D'après le témoignage de l'ancienne Inspectrice de la danse Anne-Marie Sandrini à l'issue d'une visite au Conservatoire de Région de Strasbourg en 2011, plusieurs enseignant(e)s ont vécu une dépression et ont nécessité un arrêt de travail de longue durée face à cet impératif institutionnel de suppression des repères académiques. Il y a tout lieu de s'interroger sur la visible nécessité psychologique du miroir chez les professeurs eux-mêmes: n'est-ce pas précisément parce que la danse classique requiert un retour du spectaculaire sur le kinesthésique en lequel elle se joue, contrairement aux pratiques contemporaines qui se dispensent volontiers de toute spectacularité ? Du moins ce fait impose-t-il de penser la réversibilité du spectaculaire et du kinesthésique en danse classique, de sorte qu'il ne semble plus y avoir de danse classique lorsque l'un des pôles s'en absente.

22. J'emprunte le terme de "performance » au philosophe du langage John Austin (voir note 5). De même que certains énoncés sont dits "performatifs» (de l'anglais to perform: agir et produire), de même je pose ici que la danse classique se crée à mesure qu'elle s'effectue, et qu'elle ne saurait préexister au geste qui la dessine.

23. Nous mettons entre parenthèses le préfixe «néo " pour exprimer le fait que ce que nous avançons s'applique à la danse classique autant qu'à la danse néoclassique; même s'il nous semble bien, in fine, que la danse néoclassique valide mieux encore notre propos que la danse académique originelle, parce qu'elle en est dans une certaine mesure l'aboutissement dynamique. 24. On trouve beaucoup de captations vidéos de ballets sur le net. Ici un extrait d'une répétition dudit ballet Paquita, documentaire «Carnets de la danse » diffusé sur Antenne 2 le 8 octobre 1984, réalisation de Jean-Jacques Fourgeaud, Archives de l'INA : https://www.youtube.com/watch? $\mathrm{v}=\mathrm{oDgrli50V} 2 \mathrm{~g}$, page consultée le 31 octobre 2017.

25. Le texte manuscrit est conservé à la Bibliothèque Nationale de l'Opéra de Paris. PAVLOVA Anna, Souvenirs d'une princesse de la danse, trad. Sébastien Voirol, s.l., s.n., s.d. (BNF, BibliothèqueMusée de l'Opéra, Magasin, AID-3397).

26. Notre propos est ici centré sur l'expérience en première personne de la danse classique, et non sur le geste allographique d'un chorégraphe qui ne participerait pas de l'œuvre.

27. Deux voies phénoménologiques s'opposent : la première s'intéresse au vécu immanent de l'artiste, ici l'interprète, et c'est celle pour laquelle nous optons ; elle est « charnelle », autrement dit « kinesthésique ». La seconde voie, qui n'est pas la nôtre, se préoccupe de la réception du spectacle par le spectateur. 
28. Voir HARDY Jean-Sébastien, Phénoménologie des kinesthèses et Ontologie du geste: Constitutions originaires du monde et de la chair chez Husserl, thèse de doctorat en Philosophie, sous la direction de Jean-Luc Marion, Université Paris-Sorbonne/Paris IV et Université de Laval, Québec, Canada, 2014. www.theses.ulaval.ca/2014/30866/30866.pdf.

29. Nous réitérons ici notre optique : une approche phénoménologique de la danse classique à la première personne, c'est-à-dire du point de vue du corps dansant.

30. Nous reprenons ici la distinction d'Aristote en Physique II entre la cause motrice qui initie le mouvement, et la cause finale que l'effectuation du mouvement vise à réaliser.

31. WINNICOTT Donald Woods, Jeu et Réalité (1971), trad. Fr. Claude Monod, Jean-Baptiste Pontalis, Paris, Folio Essais, 2015.

32. Voir GODARD Hubert, «Le geste et sa perception », in MICHEL Marcelle, GINOT Isabelle, La Danse au XX siècle, Paris, Éditions Bordas, 1995, pp. 224-229.

33. Voir MERLEAU-PONTY Maurice, Phénoménologie de la perception (1945), « La spatialité du corps propre et la motricité », Paris, Gallimard, 2001, pp. 114-172.

34. Voir note 5 relative au terme " performer».

35. Visible via https://www.youtube.com/watch?v=ZHU5QLEjGAg, page consultée le 31 octobre 2017.

36. BASSELIER Laetitia, "Une histoire du (néo)classique en dix ballets », revue électronique Danses avec la plume, 12 février 2015: http://www.dansesaveclaplume.com/en-coulisse/unehistoire-du-neoclassique-en-10-ballets/, page consultée le 31 octobre 2017.

37. Pensons à Serge Lifar et Maurice Béjart en France et en Suisse, à Jiř̌́ Kylián aux Pays-Bas, à l'Américain William Forsythe en Allemagne, au Suédois Mats Ek, pour n'évoquer que les piliers désormais supplantés (est-ce vraiment le terme ?) par la relève. Malgré tout, le néoclassique reste ancré en terre nord-américaine et la création chorégraphique française en classique comme en néoclassique reste très mince - Thierry Malandain, directeur du Centre Chorégraphique National de Biarritz, est un des rares chorégraphes à lui réinsuffler une certaine dynamique sur le territoire français. Benjamin Millepied, ancien Directeur de la Danse à l'Opéra de Paris, a souhaité opérer une telle transition à échelle nationale, mais l'opéra de Paris est une vieille dame et il semble préférable de «créer» des ballets néoclassiques au sein de structures d'abord indépendantes. La compagnie de Benjamin Millepied est localisée aux États-Unis.

38. Voir HUSSERL Edmund, La Crise des sciences européennes et la Phénoménologie transcendantale (1936), trad. fr. Gérard Granel, Paris, Gallimard, 2004.

39. Voir notamment Romain Bigé, «Ce que la phénoménologie peut apprendre de la danse. Straus, Merleau-Ponty, Patočka ", Recherches en danse [En ligne], 5|2016, mis en ligne le 15 décembre 2016, page consultée le 31 octobre 2017. URL: http://danse.revues.org/1394; Katharina Van Dyk, "Sentir, s'extasier, danser ", Implications philosophiques [En ligne], première partie mise en ligne le 19 juin 2010 et deuxième partie mise en ligne le 21 juin 2010, page consultée le 31 octobre 2017. URL : http://www.implications-philosophiques.org/langage-etesthetique/implications-de-la-perception/sentir-s\%E2\%80\%99extasier-danser-1/ et http:// www.implications-philosophiques.org/langage-et-esthetique/implications-de-la-perception/ sentir-s\%E2\%80\%99extasier-danser-2/ ; Paule Gioffredi, «Phénoménologie de la danse contemporaine ", Recherches en danse [En ligne], 1|2014, mis en ligne le 01 mars 2013, page consultée le 31 octobre 2017. URL : http://danse.revues.org/596. 


\section{RÉSUMÉS}

Nous nous proposons ici de penser la danse classique et néoclassique, non comme simple arrangement esthétique de figures techniques, mais comme élaboration à même la chair de l'interprète. Ainsi, en employant le syntagme "fabrique de la danse classique ", nous ancrons notre réflexion sur un socle à la fois phénoménologique et performatif. Nous revendiquons une démarche phénoménologique d'autant plus singulière que la danse académique n'a, semble-t-il, pas fait l'objet de la moindre enquête phénoménologique à ce jour. Notre projet est de mettre en évidence le caractère philosophiquement fécond d'une pratique qui ne saurait se réduire, d'un côté, à une technique, de l'autre, à un pur spectacle. Dans le même temps, nous souhaitons ouvrir la voie à une réflexion philosophique à partir de la danse classique elle-même, qui n'en fasse ni l'expression d'une spiritualité mystique, ni un rejeton anecdotique de la réflexion philosophique élaborée à partir de la danse moderne et contemporaine. À ce titre, nous postulons la singularité extrêmement féconde et actuelle d'une philosophie ancrée dans la pratique de la danse classique.

We want here to think the different kinds of ballet practice and ballet performance, not only as a mere arrangement of technical figures, but mainly as a practice elaborated from the flesh of the ballet dancer himself/herself. While using the expression «ballet fabrication », we anchor our thoughts at the same time in phenomenological philosophy and analytical philosophy of performance. We aim to highlight the fact that the ballet practice cannot be reduced, on one side, to a technique, and on another side, to romantic metaphysics. We wish to open a way of philosophical thinking from the ballet practice itself, so that such a philosophy was not considered as mystic, neither an anecdotic example of «the true " philosophy of dance made from contemporary dance. We want to assert that a philosophy anchored in the ballet practice is at the same time, very fruitful, actual, and has got its own singularity.

\section{INDEX}

Mots-clés : Chair, danse classique, danse néoclassique, fabrique, performance

Keywords : Classical ballet, fabrication, flesh, neoclassical ballet, performance

\section{AUTEUR}

\section{CHRISTINE LEROY}

Christine Leroy est agrégée de philosophie et docteure en philosophie esthétique de l'Université Paris I. Sa thèse doctorale a porté sur la phénoménologie de l'empathie kinesthésique, appliquée à l'expérience du spectateur en danse-théâtre. Tout en restant très attachée à l'approche phénoménologique, elle travaille désormais sur l'ancrage charnel des éthiques du care et est chercheuse associée au Laboratoire LEGS de Paris 8. 